\title{
Sobrevivência em Redes Ópticas
}

\author{
Helder M. N. da S. Oliveira ${ }^{1}$, Nelson L. S. da Fonseca (orientador) ${ }^{1}$ \\ ${ }^{1}$ Instituto de Computação - Universidade Estadual de Campinas (UNICAMP) \\ Campinas, SP, Brasil \\ helder@lrc.ic.unicamp.br, nfonseca@ic.unicamp.br
}

\begin{abstract}
Resumo. Neste artigo, apresentam-se os dois estudos realizados na dissertação de mestrado "Sobrevivência em Redes Ópticas". O primeiro investiga em qual camada da rede multicamada IP/MPLS sobre OTN sobre WDM, deve-se prover sobrevivência. Resultados indicam que a proteção implementada na camada OTN é mais vantajosa do que a implementada na IP/MPLS ou na camada WDM. O segundo estudo aborda sobrevivência em redes ópticas elásticas. São propostos algoritmos de sobrevivência baseados em p-cycles FIPP para uma única falha bem como para duas falhas na rede. Estes algoritmos fornecem $100 \%$ de proteção, para redes com alta conectividade.
\end{abstract}

\begin{abstract}
In this article, we present two problems investigated in the dissertation "Survivability in Optical Networks". The first answers the question on which layer of an IP/MPLS over OTN over WDM multilayer network should survivability be provided. Results indicate that protection on the OTN layer is more advantageous than either on the IP / MPLS or WDM layer. The second problem focus on survival in elastic optical networks. Algorithms based on FIPP p-cycles for single and two network failures were proposed. These algorithms can provide $100 \%$ protection for networks with high connectivity.
\end{abstract}

\section{Introdução}

A tecnologia wavelength-division multiplexing (WDM) propicia grande capacidade de transmissão para a camada de enlace da Internet, através da multiplexação de vários comprimentos de onda, em uma única fibra. Redes WDM empregam alocação de faixas do espectrum fixa por comprimento de onda e faixas menores chamadas banda de guarda para separar dois comprimentos de onda. Dada a enorme capacidade de uma fibra óptica, qualquer interrupção devido à falhas de equipamento ou ruptura de enlace implica em uma enorme perda de dados. Essa vulnerabilidade tem motivado o desenvolvimento de diferentes esquemas de restauração e proteção a falhas em redes ópticas.

Para que possa haver uma maior integração entre a camada IP e a rede óptica subjacente, propôs-se a camada Optical Transport Network (OTN). OTN oferece multiplexação e comutação eficiente de sinais de alta velocidade (cerca de $100 \mathrm{Gbps}$ ), bem como define uma camada de invólucro digital, que é vantajoso em relação as redes legadas SONET. No entanto, a introdução da camada OTN nas atuais redes IP sobre WDM demanda a superação de uma série de desafios em gerenciamento de redes, especialmente em sobrevivência multicamadas.

Em redes WDM, a capacidade de um comprimento de onda pode acomodar demandas de banda passante de diferentes magnitudes através da técnica de agregação de tráfego, porém estas não evitam à subutilização do espectro uma vez que a demanda total 
raramente coincidem com a capacidade exata de um comprimento de onda. Tal rigidez, recentemente, motivou o surgimento das redes ópticas elásticas (ou flexgrid), sendo a Multiplexação por divisão de frequências ortogonais (OFDM) a modulação mais popular nestas redes. OFDM é uma tecnologia de transmissão de multi-portadora que divide canais de alta taxa de dados em um conjunto de canais, chamados subportadoras ortogonais com baixas taxas de transmissão [Shao et al. 2012]. Apesar da existência de inúmeros métodos de proteção em redes WDM, estes não podem ser aplicados para se prover proteção em redes elásticas devido a diferença da granularidade de alocação de espectrum nessas duas redes.

A dissertação [Oliveira 2014] estudou a provisão de proteção nas atuais tecnologias de redes ópticas, introduziu dois esquemas de sobrevivência: um para arquitetura de três camadas, IP/ MPLS sobre OTN sobre WDM e outro para redes ópticas elásticas baseado em $p$-Cycle. O primeiro esquema investiga em qual camada da rede óptica multicamada mecanismo de proteção devem ser implementado. O segundo esquema utiliza p-cycle que é uma técnica de proteção utilizada em WDM, porém, cujo o uso para fornecer proteção em redes ópticas elásticas constitui um estudo original sem precedentes. Este artigo apresenta uma amostragem dos resultados númericos disponíveis em [Oliveira 2014].

\section{Trabalhos Relacionados}

Trabalhos recentes abordam aspectos de introdução da nova tecnologia de camada de transmissão OTN [Wang and Ying 2012]. O trabalho seminal em [Katib and Medhi 2011b] considera a camada OTN em uma arquitetura de três camadas. Os autores introduziram também um modelo de otimização para proteção em rede com as três camadas IP/MPLS sobre OTN sobre WDM [Katib and Medhi 2011a], bem como uma heurística para reduzir a complexidade do problema. Apresentaram, também, um estudo com base na variação de parâmetros de rede para entender o impacto desses na capacidade de proteção e no custo total da rede. No entanto, a questão de em qual nível deve-se implementar o mecanismo de proteção permaneceu em aberto.

Apesar de terem sido propostos diversos algoritmos de roteamento e alocação de espectro(routing and spectrum alocation - RSA) para redes elásticas, pouca atenção tem sido dada a questão de proteção. Foi proposto em [Pages et al. 2012] um mecanismo que atua nos transponders ociosos no nó de origem de uma requisição de enlace de alta taxa de transmissão para fragmentá-lo em vários de baixa taxa de transmissão, mais facilmente alocáveis na rede. Os autores de [Shao et al. 2012] propuseram políticas conservadoras de proteção em redes ópticas elásticas e os autores em [Patel et al. 2011] propuseram técnica de sobrevivência "transparente". Em Liu et al. [Mukherjee 2006], foi proposto uma nova técnica para a proteção que proporciona aos caminhos ópticos primários compartilhar o espectro de caminhos de backup se os caminhos primários forem disjuntos. Nenhum destes trabalhos, no entanto, utilizaram a eficiente técnica de proteção via p-cycle.

\section{Proteção em Redes Ópticas WDM Multicamda}

A dissertação [Oliveira 2014] apresenta um esquema para sobrevivência de redes em três camadas, IP/ MPLS sobre OTN sobre WDM, que protege 100\% os fluxos em cada camada contra uma falha de enlace. Nesta arquitetura, os equipamentos de alta velocidade na camada IP/MPLS, conhecidos como LSRs (Label Switching Routers), estão fisicamente conectados a comutadores ópticos (OXCs). Elaborou-se, na dissertação, um modelo de 
capacidade de rede (normal e de proteção) que considera o custo da rede e o grau de proteção em função da inserção dos mecanismos de proteção em diferentes camadas.

$\mathrm{Na}$ avaliação da adequação das diferentes camadas para hospedar os mecanismos de proteção, utilizaram-se duas topologias de rede: a topologia NSF com 14 nós e a topologia Metro com 23 nós. O desempenho da rede foi avaliado em função da capacidade normal e de proteção. Para tal, utilizou-se um modelo de programação linear inteira, implementado no software de otimização CPLEX 12.6. Foram avaliados vários parâmetros para examinar o impacto no desempenho de cada camada da rede. Para esta topologia, o número total de demandas bidirecionais é 253, assumindo uma demanda entre cada par LSR, cuja demanda média é de 5 Gbps.

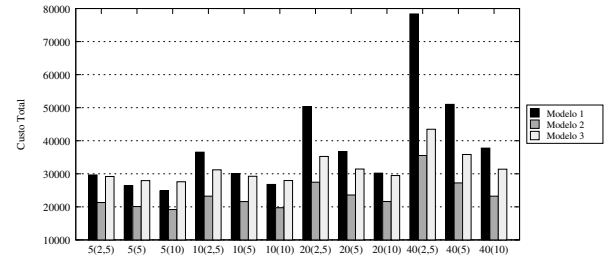

(a) Topologia NSF

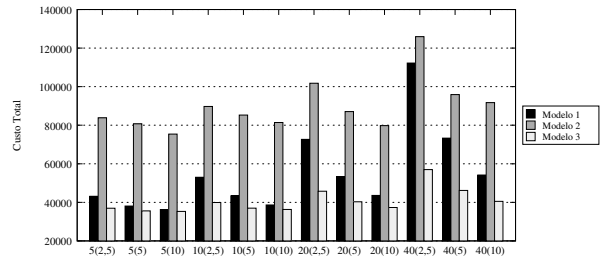

(b) Topologia Metro

Figura 1. Custo total dos três modelos para UK-cr3

A Figura 1(a) mostra uma comparação do custo para os três modelos de proteção investigados para a topologia NSF. O custo total do Modelo 1 (Proteção IP/MPLS) é influenciado tanto pelo IP-custo (custo por Gbps da camada IP) quanto pelo valor de $M$ (granularidade da camada IP/MPLS). O custo total aumenta aproximadamente $30 \%$, quando o IP-custo dobra e aumenta entre $13 \%$ a $50 \%$, quando o valor de $M$ dobra. $\mathrm{O}$ custo total do Modelo 2 (proteção na camada OTN) é menor do que o do Modelo 1, uma vez que o custo do Modelo 2 é pouco influenciado pelos valores de IP-custo e $M$. O custo total do Modelo 2 aumenta 18\%, quando IP-custo dobra e de 7 a $30 \%$, quando o valor de $M$ dobra. O custo total do Modelo 3 (Proteção na camada WDM) é influenciado tanto pelo IP-custo quanto pelo valor de $M$. O custo total do Modelo 3 aumenta, em média, $12 \%$, quando se dobra o IP-custo e entre 0,3 e $17 \%$ quando os valores de $M$ dobram. Para um IP-custo fixo, o custo total incrementa em função do valor de $M$ uma vez que um número mais elevado de maiores demandas podem ser satisfeitas. A Figura 1(b) mostra os resultados para a topologia Metro.

Para ambas as topologias, o Modelo 1 e o Modelo 3 tiveram comportamentos semelhantes. Em geral, observa-se, também, que o custo total é maior. Para ambas as topologias, a capacidade de proteção é aproximadamente o dobro da capacidade normal. O custo de fornecer proteção para as camadas OTN é altamente influenciada pela conectividade da rede. Para redes com baixa conectividade, como a rede NSF, proporcionar proteção da camada OTN é viável. No entanto, para redes com grande conectividade, o custo desse tipo de proteção é muito alto. O custo de proteção da camada OTN é mais vantajoso do que o custo de proteção nas camada IP/MPLS e WDM.

\section{Proteção em Redes Ópticas Elásticas}

O problema de roteamento e alocação de espectro (RSA) em redes ópticas elásticas engloba a restrição de continuidade de espectro presente nos problemas de roteamento e alocação de comprimentos de onda (RWA) nas redes WDM. Esta restrição impõe a 
utilização do mesmo espectro nas fibras ao longo da rota de um caminho óptico, além disso no problema RSA deve-se observar a restrição de contiguidade do espectro que determina que slots devem ser alocados de forma contínua no espectro.

O problema de roteamento e alocação de espectro é um problema NP-difícil e heurísticas são necessárias para resolver esse problema. Os algoritmos propostos nesta dissertação modelam a disponibilidade de espectro na rede como um multigrafo rotulado. Um multigrafo é um grafo que pode ter várias arestas (também chamadas de "arestas paralelas"), conectando o mesmo par de nós.

O p-cycle é um esquema de proteção no qual a capacidade reservada é pré-conectada e que forma estruturas em anel para proteção de redes em malha [Asthana et al. 2010]. A diferença fundamental entre p-cycle e proteção em anel é a proteção de enlaces transzonais, que são enlaces que não estão no anel (ciclo) porém, cujos dois nós finais estão. Um caso especial de $p$-cycle para proteção de caminho é o chamado $p$-cycle de Proteção de Caminho com Independência de Falha (FIPP). p-Cycles FIPP fornecem proteção de caminhos para caminhos com nós finais sobre o $p$-cycle.

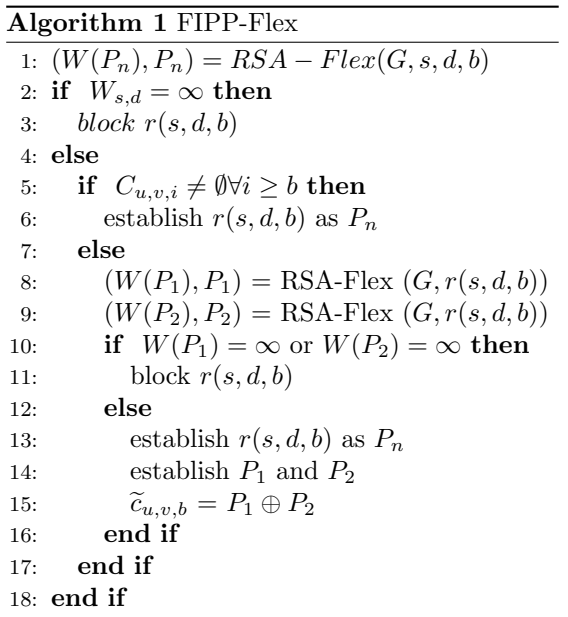

(a) Algoritmo

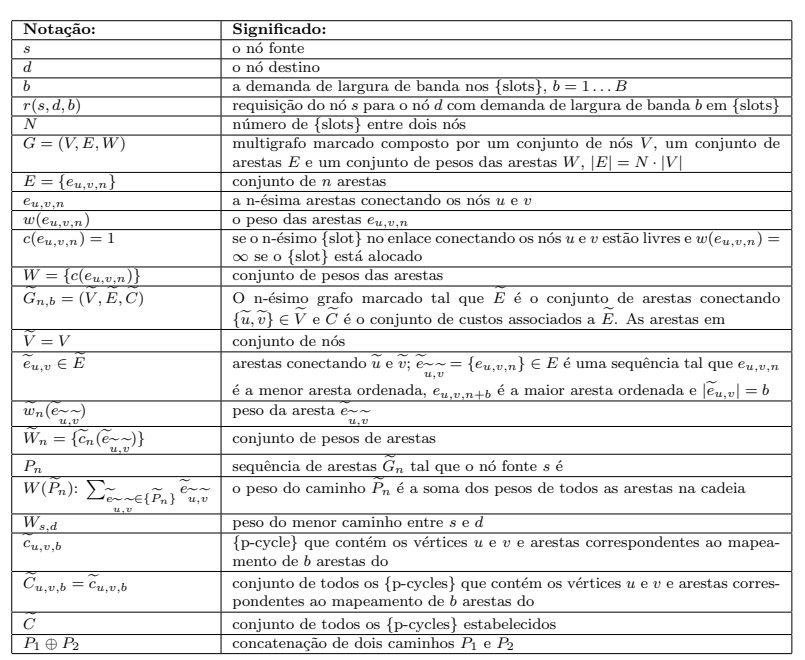

(b) Notação

Figura 2. Algoritmo FIPP e Notação

Para avaliar o desempenho do algoritmo proposto, o simulador FlexGridSim foi utilizado nas simulações. Em cada simulação, 100.000 requisições foram geradas e para cada algoritmo foi utilizado o mesmo conjunto de sementes. Utilizou-se o metodo de replicação independente e adotou-se nível de confiança de $95 \%$ para os intervalos de confiança. Na rede elástica simulada, o espectro foi dividido em 240 slots de $12,5 \mathrm{GHz}$ cada, com requisições distribuídas uniformemente entre os pares de fontes e destinos. Os algoritmos introduzidos na dissertação chamados FIPP-Flex e FIPP-Flex-twofailure, resolvem o problema de estabelecimento de caminhos ópticos em redes protegidas por p-cycles FIPP tolerantes respectivamente, a uma e a duas falhas. Nesses algoritmos, caminhos ópticos são estabelecidos se e somente se puderem ser protegidos por um $p$ cycle FIPP, que protege somente caminhos primários disjuntos. Requisições para estabelecimento de caminhos ópticos chegam dinamicamente e para cada requisição tentase associar um $p$-cycle existente para proteger o caminho óptico requisitado. Caso não haja $p$-cycle que possa proteger o caminho óptico, então, cria-se um novo $p$-cycle para a 
requisição. Se nenhum caminho for encontrado para proteger o caminho solicitado, então este não é estabelecido. O algoritmo FIPP-Flex garante um caminho de proteção para cada caminho óptico estabelecido e a proteção é garantida para falhas únicas. A Figura 2 descreve o algoritmo. O algoritmo FIPP-Flex-twofailure diferentemente do algoritmo FIPP-Flex garante um caminho de proteção totalmente disjunto do caminho primário, e garante assim proteção para duas falhas simultâneas.

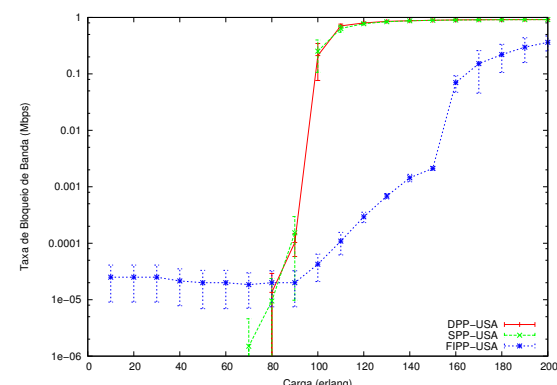

(a) Topologia USA

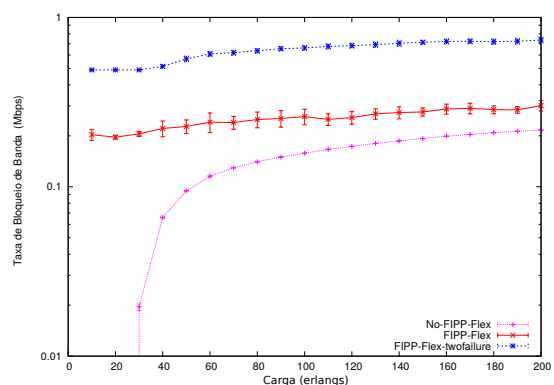

(b) Topologia DFN

Figura 3. Bloqueio de banda em função da carga da rede

A Figura 3(a) mostra a razão entre banda bloqueada e solicitada (Bandwith Blocking Ratio, BBR) em função da carga para a topologia USA. As curvas SPP [Patel et al. 2011] e DPP [Shao et al. 2012] mostram resultados de algoritmos de proteção existentes na literatura e a curva FIPP representa o algoritmo FIPP-Flex. As curvas SPP e DPP produzem valores similares de BBR. Observa-se que quando a rede satura a BBR atinge seu valor máximo. O algoritmo FIPP capitaliza na alta conectividade a topologia USA e o BBR aumenta suavemente em função do aumento da carga. Os valores de BBR produzidos pelo FIPP-Flex são menores do que os produzidos pelos algoritmos SPP e DPP após a saturação rede.

A Figura 3(b) apresenta a BBR em função da carga para redes sem proteção (NoFIPP-Flex), com proteção contra uma falha (FIPP-Flex) e com proteção contra duas falhas (FIPP-Flex-twofailure), para a topologia DFN. O valor da BBR para proteção contra duas falhas está em torno de 0,5 devido a exigência de se ter três caminhos disjunto o que faz com que alguns nós da rede sejam sobrecarregados mais rapidamente enquanto que o valor da BBR gerada para se proteger uma única falha está em torno de 0,3. Observase que a partir da carga de 50 erlangs, o impacto no BBR para se proteger contra uma única falha é de aproximadamente 0,2 , ou seja, bloqueia-se apenas $20 \%$ a mais da banda requisitada do que em uma rede que não provê qualquer proteção de caminho.

\section{Trabalhos publicados}

Os resultados da dissertação [Oliveira 2014] foram relatados nos artigos [Oliveira and da Fonseca 2014b][Oliveira and da Fonseca 2014a], publicados no SBRC 2014 (Qualis B2) e IEEE GLOBECOM 2014 (Qualis A1). Além disso, submeteu-se artigo para a conferencia IEEE GLOBECOM 2015 e encontram-se em elaboração artigos para submissão a periódicos.

\section{Conclusões}

Neste artigo, foram apresentados as contribuições da dissertação [Oliveira 2014]. Investigou-se o esquema de proteção em redes ópticas WDM e proteção em redes ópticas 
elásticas. Introduziu-se um modelo de projeto de proteção de rede para uma rede de três camadas IP/MPLS sobre OTN sobre WDM. Nesta arquitetura, o projeto de sobrevivência oferece proteção apenas para a capacidade normal de cada camada a fim de minimizar os recursos de proteção. Estudou-se a influência de proteção em cada camada, variando-se diferentes parâmetros. Concluiu-se que é vantajoso proteger a camada intermediária OTN pois minimiza o custo e a capacidade requerida.

Algoritmos para a criação de caminhos ópticos em redes elásticas protegidos por p-cycles FIPP para uma falha bem como para falhas duplas foram, também, introduzidos na dissertação. O algoritmo FIPP-Flex fornece $100 \%$ de proteção para falhas individuais enquanto o algoritmo FIPP-Flex-twofailure provê $100 \%$ de proteção contra duas falhas. As contribuições da dissertação são originais e avançam o estado da arte em sobrevivência de redes ópticas modernas.

\section{Referências}

Asthana, R., Singh, Y., and Grover, W. (2010). p-cycles: An overview. IEEE, Communications Surveys Tutorials, 12(1):97 -111.

Katib, I. and Medhi, D. (2011a). A network protection design model and a study of threelayer networks with ip/mpls, otn, and dwdm. In Design of Reliable Communication Networks (DRCN), 2011 8th International Workshop on the, pages 17-24.

Katib, I. and Medhi, D. (2011b). A study on layer correlation effects through a multilayer network optimization problem. In Teletraffic Congress (ITC), 2011 23rd International, pages $31-38$.

Mukherjee, B. (2006). Optical wdm networks. New York: Springer.

Oliveira, H. and da Fonseca, N. (2014a). Algorithm for fipp p-cycle path protection in flexgrid networks. In Global Communications Conference (GLOBECOM), 2014 IEEE, pages $1278-1283$.

Oliveira, H. and da Fonseca, N. (2014b). Protection in elastic optical networks against up to two failures based fipp p-cycle. In Computer Networks and Distributed Systems (SBRC), 2014 Brazilian Symposium on, pages 369-375.

Oliveira, H. M. N. S. (2014). Sobrevivencia em redes opticas. Master's thesis, University of Campinas.

Pages, A., Perello, J., and Spadaro, S. (2012). Lightpath fragmentation for efficient spectrum utilization in dynamic elastic optical networks. In Optical Network Design and Modeling (ONDM), 2012 16th International Conference on, pages 1-6.

Patel, A., Ji, P., Jue, J., and Wang, T. (2011). Survivable transparent flexible optical wdm (fwdm) networks. In Optical Fiber Communication Conference and Exposition (OFC/NFOEC), 2011 and the National Fiber Optic Engineers Conference, pages 1-3.

Shao, X., Yeo, Y.-K., Xu, Z., Cheng, X., and Zhou, L. (2012). Shared-path protection in ofdm-based optical networks with elastic bandwidth allocation. In Optical Fiber Communication Conference and Exposition (OFC/NFOEC), 2012 and the National Fiber Optic Engineers Conference, pages 1-3.

Wang, Q. and Ying, G. (2012). Otn for the future transmission network. In Photonics and Optoelectronics (SOPO), 2012 Symposium on, pages 1-4. 